\title{
Glossaire Microbiote
}

ARN ribosomiques (ARNr) 16S: ARN constituant la petite sous-unité des ribosomes des procaryotes. Les gènes codant ces ARN sont appelés ADNr 16S.

Auxotrophie : un organisme auxotrophe pour un nutriment est un organisme auquel il faut fournir le nutriment qu'il ne peut synthétiser. Axéniques : les animaux axéniques sont utilisés pour l'étude de l'influence du microbiote sur la physiologie de l'hôte. Ces animaux, dont le tube digestif ne contient aucun microorganisme, sont prélevés stérilement à la naissance (par césarienne) et donc non colonisés et élevés à l'abri de toute contamination microbienne (en anglais, germfree).

Bactéries filamenteuses segmentées (en anglais segmented filamentous bacteria, SFB) : les bactéries filamenteuses segmentées sont des bactéries de la famille des Clostridiales qui colonisent l'intestin de nombreuses espèces, dont la souris et probablement aussi l'homme, sans provoquer de maladies; elles vivent en symbiose avec les cellules épithéliales et sont nécessaires à la maturation de la barrière immune intestinale.

Commensalisme : type de relation où une espèce en exploite une autre sans notion de dangerosité.

Dextran sulfate sodium (DSS, en français, dextran sulfate de sodium) : le DSS est communément utilisé pour induire une colite dans le modèle murin de $\mathrm{MICl}$ (voir MICI).

Dysbiose : déséquilibre du microbiote (en qualité et/ou quantité) (voir Eubiose).

Entérotype : signature bactérienne intestinale. II s'agit de l'ensemble des bactéries constituant un microbiote intestinal particulier. Un entérotype peut être associé à une pathologie.

Eubiose : état d'un microbiote considéré à l'équilibre, «normal », présumé remplir toutes les conditions pour faire bénéficier de ses effets la physiologie de l'hôte (métabolisme, immunité, effet barrière).

Gnotoxénique : animal axénique auquel on a inoculé une ou plusieurs souches microbiennes définies (voir Axéniques).

M cells ou cellules M (microfold cells) : cellules situées dans l'épithélium intestinal au sein des plaques de Peyer (voir Plaques de Peyer). Elles diffèrent des cellules de l'épithélium par l'absence de bordure en brosse. Elles incorporent par endocytose les antigènes endoluminaux et les transfèrent aux cellules dendritiques qui les présentent aux lymphocytes $B$.

Métagénomique : étude globale des gènes d'une population, sans les détailler individu par individu $[1,2](\rightarrow)$.

$\mathrm{MICl}$ : maladies inflammatoires chroniques de l'intestin. Elles regroupent principalement la maladie de Crohn

(MC) et la rectocolite hémorragique $(\mathrm{RCH})$, toutes deux inflammatoires mais touchant des segments distincts de

l'appareil digestif et d'évolution différente.

Microbiotes : communautés microbiennes colonisant différents sites. On parle de microbiote intestinal, pulmo-

naire ou cutané.

Microbiome : génome des microbes formant le microbiote déterminé de façon globale [1] $(\rightarrow)$.

Mutualisme : interaction entre deux espèces, dans laquelle les organismes impliqués tirent tous les deux profit de cette relation.

Pathobionte : membre opportuniste normalement sous-dominant de la flore commensale.

Pathovar : groupes clonaux au sein d'une espèce bactérienne produisant des facteurs de virulence faisant défaut chez les souches commensales.

$(\rightarrow)$ Voir également les Synthèse de J. Weissenbach et A. Sghir, et de H.M. Blottière et J. Doré, pages 937 et 944 de ce numéro

Plaques de Peyer : elles sont formées d'un épithélium intestinal particulier, recouvrant un ou plusieurs follicules lymphoïdes, constitués de lymphocytes B organisés en un centre germinatif, de lymphocytes T formant une couronne entourant ce centre germinatif, de macrophages et de cellules dendritiques présentant les antigènes.

Prébiotique : composant non digestible par l'hôte qui stimule de manière sélective au niveau du côlon la multiplication ou l'activité d'un ou d'un nombre limité de groupes bactériens susceptibles d'améliorer la santé de l'hôte.

Probiotique : microorganisme vivant (bactérie ou levure) partenaire de l'intestin qui a un effet bénéfique en améliorant l'équilibre du microbiote.

Ribotypage : analyse des ARN ribosomiques.

Ribotype : la séquence exacte des ARN ribosomiques permet de définir une empreinte spécifique de l'espèce, le ribotype.

Taxonogenomics : études incluant des données phénotypiques (taxonomie) et le séquençage du génome.

spp. : abréviation de « espèces ». Exemple : Clostridium spp. signifie les différentes espèces de Clostridium.

Syntrophie : relation mutualiste établie entre deux espèces au cours de laquelle chaque espèce produit un élément nutritif essentiel au développement de l'autre.

Symbiose : association intime et durable entre deux organismes hétérospécifiques, et parfois plus de deux. Les organismes sont qualifiés de symbiotes ou plus rarement symbiontes.

Xénobiotiques : substances étrangères à l'organisme de faible masse moléculaire (tels que médicaments, polluants, aliments).

\section{RÉFÉRENCES}

1. Weissenbach J, Sghir A. Microbiotes et métagénomique. Med Sci (Paris) 2016 ; 32 : 937-43.

2. Blottière HM, Doré J. Impact des nouveaux outils de métagénomique sur notre connaissance du microbiote intestinal et de son rôle en santé humaine : enjeux diagnostiques et thérapeutiques. Med Sci (Paris) 2016 ; 32 : 944-51. 